International Journal of Applied Dental Sciences 2021; 7(2): 106-110

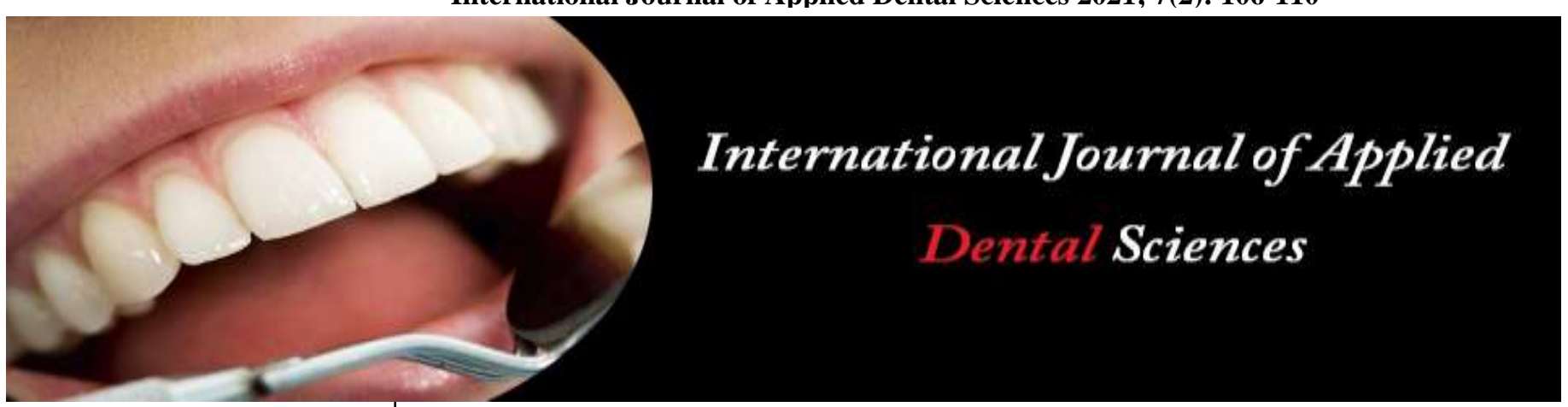

ISSN Print: 2394-7489

ISSN Online: 2394-7497

IJADS 2021; 7(2): 106-110

(C) 2021 IJADS

www.oraljournal.com

Received: 21-01-2021

Accepted: 23-02-2021

Dr. Shruti Balasubramanian Post Graduate, Pediatric \& Preventive Dentistry, Government Dental College \& Hospital, Medical College Premises, Medical Square Nagpur, Maharashtra, India

Dr. Ritesh Kalaskar Professor, Pediatric \& Preventive Dentistry, Government Dental College \& Hospital, Medical College Premises, Medical Square Nagpur, Maharashtra, India

Dr. Ashita Kalaskar Associate Professor, Oral Medicine and Radiology Government Dental College \& Hospital, Medical College Premises, Medical Square Nagpur, Maharashtra, India

Corresponding Author: Dr. Shruti Balasubramanian Post Graduate, Pediatric \& Preventive Dentistry, Government Dental College \& Hospital, Medical College Premises, Medical Square Nagpur, Maharashtra, India

\section{The new normal of paediatric dental practice post COVID-19: A review}

\author{
Dr. Shruti Balasubramanian, Dr. Ritesh Kalaskar and Dr. Ashita Kalaskar
}

DOI: https://doi.org/10.22271/oral.2021.v7.i2b.1197

\section{Abstract}

Background: Novel coronavirus disease (COVID-19) is one of the worst pandemics known to mankind. It affects all the age groups but children present with milder symptoms and may also be asymptomatic. In the current scenario paediatric dentists pose high risk of the disease due to close contact with not just children but also with their parents. Additionally, majority of paediatric dental procedures advocate the use of handpiece which generates immense aerosol increasing the transmission risk by manifold.

Objective: This review aims to highlight the transmission of COVID-19 in dental setting, its impact on paediatric dental setting and dental education and the new normal of paediatric dental practice.

Modification in Dental Practice: With health authorities predicting that the novel coronavirus may become an endemic in the community, it necessitates modification in dental practice to adapt to the new normal. It requires modification in behaviour management techniques, tele-triaging of patients, stringent infection control, minimising AGPs and replacing them with minimally invasive techniques along with use of adequate isolation. The ongoing pandemic has also severely impacted the dental education therefore advocating modifications in the same with the advent of online teaching and webinars.

Conclusion: Paediatric dentists are at high risk of acquiring the infection, hence it is the need of the hour to adapt to the new normal of practice at the earliest. We are an important part of the community; hence it is our foremost duty to contain the spread of this virus and not act as an aid in its transmission.

Keywords: COVID-19, pediatric dentistry, review

\section{Introduction}

The emergence and escalated spread of novel coronavirus disease (COVID-19) captured the attention of entire world and led to its declaration as a global pandemic by World Health Organisation (WHO) on March, 11, $2020^{[1]}$. It is considered as one of the worst pandemics known to affect the mankind since the Spanish Flu in 1918-1919 [2].

Coronavirus disease has been observed to affect all age groups ranging from paediatric to geriatric patients. Its clinical presentation could be asymptomatic or a range of respiratory symptoms including sore throat, cough, pyrexia, malaise and dyspnoea. Apart from these, vague symptoms like anosmia, nausea, vomiting and diarrhoea may also be present ${ }^{[3]}$.

The first confirmed paediatric case of SARS-CoV-2 infection was reported in Shenzhen on January, 20, 2020 ${ }^{[4]}$. The clinical characteristics of the disease are yet to be collated in children. Paediatric COVID-19 infection is reported to be milder compared to adults and has a better prognosis with mortality limited to the ones with co-morbidities ${ }^{[5,6]}$. Significant proportion of infected children also appear to be asymptomatic. Two studies conducted by Lu et al. ${ }^{[7]}$ and Dong et al. ${ }^{[8]}$ showed asymptomatic presentation in $15.8 \%$ and $38.8 \%$ children respectively. Another cause of concern among children is the less suggestive clinical history compared to adults which adds to the uncertainty of their infection status.

Due to the aforementioned features, there is a high tendency to underestimate the true rate of COVID-19 infection in children. This prompts greater vigilance towards children as they may function as silent carriers and play a major role in community based viral transmission owing to their asymptomatic presentation.

COVID-19 is known to transmit between close contacts hence oral healthcare professionals are reported to be at high risk due to the inherent nature of close contact while treating patients. These factors highlight the increased tendency of paediatric dentists to be affected by COVID- 
COVID-19. The first case of COVID-19 in dentist was reported on 23 January 2020 at the Department of Preventive Dentistry in the Wuhan University Dental Hospital which eventually transmitted to eight other oral healthcare professionals ${ }^{[9]}$.

This review aims to highlight the transmission of COVID-19 in dental setting, its impact on paediatric dental setting and dental education and the new normal of paediatric dental practice.

\section{Transmission of Covid-19 in Pediatric Dental Practice}

Paediatric dental practice comprises of four components i.e. dentist, patient, parents and dental assistant unlike other dental specialities. This aspect adds to the high risk of infectivity due to increase in number of people present in the compact dental operatory at a given point of time. At the present stage of the pandemic, all paediatric patients as well as their parents should be considered as potential carriers. The common route of transmission of novel coronavirus is through either direct or indirect contact, through saliva and coarse droplets [10]. Dental settings carry increased risk of coronavirus infection owing to the inherent specificity of procedures which involve face to face communication with patients, frequent contact with body fluids namely saliva, blood and handling of sharp instruments.

The airborne spread of SARS-CoV-2 is well established in literature. Majority of procedures in paediatric dentistry require the use of high-speed handpiece which causes immense aerosol generation and droplets mixed with patient's saliva and blood [11]. Researchers have established that particle size of droplets and aerosols are small enough to stay airborne for prolonged duration of time. Additionally, the relative humidity of dental clinic is high which also leads to increased tendency for virus to stay for extended period. Apart from the airborne spread, another crucial mechanism of spread is via fomite. There are numerous surfaces in a dental clinic which are frequently touched by the patients and could aid in transmission. This factor is more of a concern with children as it is difficult to control their actions. The novel coronavirus is known to persist up to a couple of days on surfaces like plastic, metal and glass. ${ }^{12}$ Hence, door knobs, dental chair handles, light, switches, tables etc. which are frequently contacted are potential source of transmission.

\section{Economic Impact of Pandemic on Dentists}

The nationwide lockdown has hugely impacted the financial situation of dental professionals. Majority of dentists in India are into private practice with burden of loan and EMI whose repayment entirely depends on the returns obtained from their practice. In April 2020, an extensive survey was conducted for over 19,000 dental professionals by ADA health policy to understand the economic impact of first wave of COVID-19. ${ }^{[13]}$ Majority of respondents were private practitioners whose clinics were closed and only seeing emergency cases. They reported that patient volume was less than $25 \%$ of what is typically seen and $45 \%$ practitioners were paying their staff partially whereas $28 \%$ were not paying them at all.

\section{Summary of Guidelines for Dental Practice}

To our surprise, at present there are no existing universal guidelines for provision of dental treatment during COVID-19 pandemic or for that matter during any epidemic.

\section{Guidelines in India}

Dental Council of India (DCI) issued unified guidelines to address the dental services in the country which would cover health care workers who are required to attend dental ailments in remote locations in the government sector, dental surgeons working in primary health centers/ small towns and different locations, those working in government and private hospital set up and practitioners working in cities with solo or multispecialty practices ${ }^{[14]}$. Basic guidelines were issued based on the categorization of locations based on red, orange and green zone ${ }^{[15]}$. They instructed closure of dental clinics in containment zones with continuation of tele-triage. Clinics in the red zone could provide emergency care whereas clinics in the orange and green zone can provide emergency as well as urgent care. They instructed on deferring routine and elective dental procedures for a later review until issue of fresh guidelines. They recommended primary triage to focus on triple A concept: Advice, Analgesics, Antimicrobials (where appropriate).

DCI categorized dental treatments into emergency and urgent procedures. Dental conditions which pose risk to patient's life are categorized as "Emergency" and procedures requiring priority care but do not pose risk to life are categorized as "Urgent".

Emergency and Urgent care for pediatric patients are enlisted below as per DCI recommendations:

\section{Emergency dental care}

- Fast spreading infections of facial spaces, Ludwig angina, acute cellulitis of dental origin and acute trismus

- Uncontrolled bleeding of dental origin

- Severe uncontrolled dental pain, not responding to routine measures

- Trauma involving the face or facial bones

- Radiographs like PNS, OPG, CBCT in facial trauma and in medico-legal situations

\section{Urgent dental care for children and adolescent}

- Acute pulpitis

- Dental abscess

- Dentoalveolar trauma

- Pain of cavitation needing temporization

- Unavoidable Dental Extractions

- Orthodontic procedures - Orthodontic appliances impinging oral mucosa, orthodontic treatment causing iatrogenic effects, delivery of clear aligners and patients on skeletal anchorage.

\section{Guidelines issued by International authorities}

Numerous recommendations were issued for provision of dental care during this pandemic by various authorities namely American dental association (ADA), Centers for disease control and prevention (CDC), USA, The ministry of health and dental council New Zealand (NZMOH), Australian dental association (AusDA), Scottish dental clinical effectiveness program (SDCEP), Royal college of surgeons of England (RCS), American Academy of Pediatric Dentistry (AAPD) ${ }^{[3]}$. Majority of international authorities also provided a case definition of emergency and urgent dental care. Emergency dental care was suggested for conditions like fever associated with pulpal and periapical infections, facial swelling, uncontrolled bleeding, facial trauma compromising airway, dentoalveolar injuries like avulsion of permanent teeth and acute dental infections likely to exacerbate systemic conditions.

These authorities have advised management of emergency and urgent dental conditions. Invasive emergency treatment 
should be deferred wherever possible and aerosol generating procedures (AGPs) should also be avoided as far as possible and replaced maximally with minimally invasive or atraumatic restorative treatment (ART). However, all the guidelines encourage the use of tele-dentistry as far as possible which includes interviewing patient via telephone, text monitoring system or video conference prior to visit and provide advice, analgesics or antimicrobials wherever appropriate.

Besides the aforementioned guidelines, we would recommend inclusion of cleft care in emergency treatment. Infants born with cleft lip and palate have an inherent difficulty in sucking of milk which leads to nasal regurgitation causing aspiration pneumonia which may lead to their death. Hence, provision of feeding plate/ obturator is extremely necessary and should be done at the earliest to aid in comfortable feeding of these infants and avert any life-threatening complications.

\section{The New Normal In Pediatric Dental Practice: Changing Perspective}

In the beginning of March 2020, New York Times had enlisted professions with high risk of infection and had mentioned dentists as number one. This explains the gravity of the situation and how essential it is for us as healthcare professionals to protect us, our families and our patients. Looking at the current increasing trend in COVID-19 cases, it does not appear that this pandemic will end anytime soon. Recently, the second wave of COVID-19 has begun globally affecting several countries and the same is predicted in India from December 2020. The lesson learnt from the 1918-1919 Spanish influenza was that it also followed a similar trajectory and was followed by a far deadlier second and third wave of infection. The factors responsible for the resurgence of cases was reckless behaviour, premature and untimely relaxation in precautionary measures by individuals as well as the government. World Health Organisation (WHO) also feared about this virus becoming just another endemic virus in our community and may never go away. Therefore, if these speculations are to be believed, dental practice will have to reorganize itself using innovative solutions to continue provision of dental care with minimal risk of cross infection. This requires us to amend our practice based on the guidelines issued by various authorities keeping in mind the best of our patient's interest and also how far is it practical to implement them.

\section{Tele dentistry}

Keeping in mind the ongoing circumstances of COVID-19, the main aim stands to minimize person to person contact as far as possible. One of the best methods to achieve this presently is adopting tele dentistry which caters to the key aspect of social distancing. It has been universally advocated by health authorities to contain the spread of SARS-CoV-2. It offers a wide range of applications thereby avoiding unnecessary visits to the dental clinic. It consists of subunits namely teleconsultation, tele diagnosis, tele triage and telemonitoring ${ }^{[16]}$. Teleconsultation is the most common form of tele dentistry which allows patients to seek advice from dentists through forms of telecommunication. Majority of the dental lesions are often evident directly hence tele diagnosis can be made with the help of dental photography thus reducing the need for close clinical examination. Machado et al. [17] enumerated the use of WhatsApp telemedicine in differential diagnosis of oral lesions during this pandemic. Another important concept in tele dentistry is the use of tele triaging which allows timely and appropriate disposition of patient symptoms via smartphone. Additionally, telemonitoring allows for regular follow up of patients by replacing physical visits with virtual visits for monitoring progression of disease and treatment outcomes. Lastly, telecommunication also allows to enquire patients regarding any history of travel or symptoms indicative of COVID-19 and also allows scheduling of appointments to permit less crowd in the waiting area.

\section{Use of personal protective equipment (PPE)}

These protective gears are designed to safeguard healthcare workers by minimizing exposure to a biological agent. These protective gears are donned based on the risk of the procedure performed mainly for aerosol generating procedures (AGPs). It comprises of head cover, face shield, goggles, masks, gloves, coverall/gowns, shoe cover. It is necessary for dentists and dental assistants to be adequately trained regarding the use of every component and proper donning and doffing of the PPE. It is necessary to remove the PPE in an appropriate manner since incorrect method leads to acquiring of infection and also its spread. It is mandatory to sanitize hands before donning and after doffing every component of the PPE. Additionally, it is necessary to properly dispose the PPE and also appropriate storage of the reusable components of PPE like N-95 masks if needed should be done. In order to permit reuse of N-95 mask, it is advisable to wear triple layered surgical masks over N-95 masks which prevents the N-95 mask from getting contaminated. All India Institute of Medical Sciences (AIIMS), New Delhi issued a protocol on reuse of N-95 masks wherein each mask could be reused on every $5^{\text {th }}$ day for up to 4 times. This reuse should be done only if masks are not visibly soiled or moist and stored in clean zip lock pouches. Certain protective gears like masks need to be mandatorily worn by all the staff members in the clinic. The use of protective gear does not replace the use of proper hand hygiene protocol and maintenance of respiratory etiquette.

However, the use of PPE poses a peculiar challenge for paediatric dentists since it instils great deal of anxiety among children making behaviour management difficult. Anxious children tend to cry which initiates cough causing spatter production thereby increasing risk of cross infection. Paediatric dentists need to work to overcome these challenges. As far as possible, it is advised to don the PPE while the child is watching and attempt should be made to make the child understand its use in simple language. Another suggestion could be to put on sticker with dentist's photograph on the PPE. Keeping the child's anxiety in mind, attempts have been made to create attractive face shields with cartoon characters using 3D printing.

\section{Behaviour management}

Behaviour management is the cornerstone of paediatric dentistry. Apart from the aforementioned challenge, there are other obstacles to managing children in the current situation. Firstly, parental presence in the operatory is not advised in order to prevent crowding in compact places. Hence, children need to be adequately trained to cooperate in the absence of parents. It is advocated to perform maximum treatments in single appointment based on child's cooperation to minimize their visits. Children who are extremely uncooperative or require protective stabilisation should be postponed for treatment as far as practically viable. 


\section{Patient entry and discharge protocol}

Firstly, patient appointments need to be scheduled telephonically to minimize crowding in the waiting room. As far as possible patient should be accompanied with single parent and their entry should be permitted only with masks. Parents should be encouraged to download Arogya Setu app to determine their risk status. Apart from obtaining history of symptoms indicative of Severe Acute Respiratory Illness (SARI) telephonically, same should be enquired in the front desk of clinic and temperature to be monitored using infrared thermal scanner. On entry, patients should be asked to sanitize their hands and instructed not to touch any surface as far as possible. On seating the patient on dental chair, clinical examination to be performed wearing double gloves, triple layer masks and face shield. Patients are advised to perform pre-procedural rinse with $10 \mathrm{ml}$ of $0.5 \%$ Povidone iodine solution. Chlorhexidine rinses which are regularly used as a pre-procedural rinse is not recommended with coronavirus since they lack oxidative potential hence considered ineffective. As far as possible non aerosol generating procedures should be performed.

While discharging the patient, payments should be encouraged digitally and any cash handling should be avoided.

\section{Modification in dental clinic setup}

To be honest, no actual modifications are required just in view of the COVID-19 since these requisites should have always been present in the dental clinic. But however, prior to this pandemic few of these aspects were neglected which have become mandatory in view of the current situation. Adequate ventilation in the operatory and frequent servicing of air conditioning system should be ensured. Indoor portable air cleaning system may be equipped with HEPA filter and UV light system. Patient's entry into the playroom should be limited and toys need to be placed at a certain distance. Chairs need to be placed at a distance of minimum 1 metre and also signage boards indicating symptoms of COVID-19 and its preventive strategies can be placed in waiting area. In clinics with more than one dental chair in a single operatory, proper distance between them should be ensured and as far as possible procedure should be performed only on single chair. Changing area should be made available for all staff and dedicated area should be kept for donning and doffing of PPE. Sufficient and dedicated space for storage of PPE, sterilised instruments and disinfectants should be ensured. Washrooms should be installed with either sensor taps or taps with elbow handle and automatic sanitiser dispenser.

\section{Disinfection of clinics}

Stringent infection control practices are the need of the hour in these trying times. After completion of treatment, the instruments should be cleared away immediately for rinsing followed by sterilization or disinfection. Three in one syringe, water lines, suction pipes should be flushed with disinfectant. After every patient, the dental chair and other contacted surfaces should be disinfected using $1 \%$ sodium hypochlorite. Fumigation should be performed at the end of the day in the dental operatory and at least twice a week in the non-clinical areas. Another alternative is the use of fogging which is commercially available $11 \%(\mathrm{w} / \mathrm{v})$ hydrogen peroxide which is stabilised by $0.01 \%$ silver nitrate.

Protocol for health workers on reaching home

It is advisable to wear scrubs in the operatory and change just prior to leaving the clinic to regular clothes. Hand washing should be done thoroughly before leaving the clinic. On reaching home, all personal belongings should be kept in a box outside which should be sanitized. Shoes should be removed outside and hands to be sanitized before entering the house. It is highly recommended to not touch anything or come in contact with family members before taking a shower which should be done immediately after entering the house.

\section{New normal in the therapeutic approaches}

It is a well-established fact that prevention is better than cure and is much cheaper and stable. But apart from this, in view of the current situation preventive strategies have gained much importance and it should be directed towards a more patient centred approach.

In 2016, European academy of paediatric dentistry documented policy pertaining to management of early carious lesion in children and young adults. ${ }^{18}$ They concluded that non-cavitated lesions can be managed non-invasively in majority of cases by incorporating a low cariogenic diet, appropriate management of the biofilm, home as well as dental office application of fluorides and sealing techniques.

The main aim of management on carious lesions is to minimize aerosol generating procedures (AGPs) as far as possible. Biological treatment alternatives for asymptomatic teeth and teeth showing signs of reversible pulpitis range from atraumatic restorative technique (ART), interim therapeutic restorations (ITR), indirect pulp therapy (IPT), hall technique (HT) or use of silver diamine fluoride (SDF). BaniHani et al. ${ }^{[19]}$ evaluated the outcomes of both biologic methods of caries management comprising of HT and IPT and conventional method of complete caries removal or pulpotomies and concluded that both were equally successful in managing carious lesions in primary teeth.

SDF has become a game changer in the current pandemic. Numerous systematic reviews substantiate the efficacy of SDF in managing carious lesion in young children and those with special health care needs ${ }^{[20]}$. It arrests caries by hampering bacterial metabolism due to presence of $\mathrm{Ag}$ and by enhancing remineralization. ADA recommends using SDF to arrest advanced cavitated carious lesions on any coronal surface of primary and permanent teeth. They suggest clinicians to prioritise the use of $38 \%$ SDF solution (biannual application) over 5\% NaF varnish. However, its biggest drawback is the blackish discolouration resulting in unesthetic appearance and metallic taste which makes it unacceptable. But it has been observed that restoration with glass ionomer cement (GIC) and composites completely masks the black discolouration.

In few cases the use of handpiece is inevitable, hence use of micromotor or anti-retraction handpiece is advocated in such scenarios. Additionally, use of rubber dam isolation and suction is mandatory for any type of procedure. Certain practitioners have also explored the use of extraoral suction device since it aids in removing toxic products and prevent cross-contamination among the patient and dentist and also works as an air sterilizer in the operatory.

\section{Challenges in Dental Education}

The current scenario has taken a major toll on both undergraduate and postgraduate teaching curriculum. During this pandemic, it is highly encouraged to continue the online lectures and seminars with the use of existing smart devices and applications which has made it possible for students to review lectures whenever and wherever possible. ${ }^{21}$ 
Additionally, patient based learning and practical procedures have taken a huge setback for students. Case studies and problem-based learning on online platform need to be encouraged. With the advent of 3D printing technology, it is possible to print 3D face, tooth, dental arch etc. to provide practical learning to the student with the sense of performing it on real patients. These changes could be adapted to continue the curriculum for students and prevent the impact of the current situation on their future.

\section{Conclusion}

Keeping in mind the unique environment of the dental clinic, the risk of COVID-19 transmission is very high. With the continuing increase in COVID-19 cases, it is viable for paediatric dentists to be well informed regarding its signs and symptoms and follow stringent infection control protocol considering every patient as potentially infected with virus. Guidelines issued by authorities cannot cover all the possible clinical situations, hence exercising professional judgement becomes crucial on whether to provide treatment or not based on the circumstances. It is viable for paediatric dentists to adapt to the new normal and utilise opportunities to encourage preventive approaches for dental diseases. It is highly recommended to minimize or attempt to eliminate aerosol generating procedures in this pandemic and even after ease of restrictions in the future. Attempts should be made to replace them with more biological and minimally invasive options.

\section{References}

1. World Health Organization https://www.who.int/newsroom/detail/27-04-2020-who-timeline---covid-19. Accessed May 31,2020.

2. Snyder MR, Ravi SJ. 1818, 1918, 2018: Two Centuries of Pandemics. Health Secur. 2018;16(6):410-5.

3. Al-Halabi M, Salami A, Alnuaimi E, Kowash M, Hussein I. Assessment of paediatric dental guidelines and caries management alternatives in the post COVID-19 period. A critical review and clinical recommendations [published online ahead of print, 2020 Jun 16]. Eur Arch Paediatr Dent. 2020, 1-14.

4. Chan JF, Yuan S, Kok KH et al. A familial cluster of pneumonia associated with the 2019 novel coronavirus indicating person-to-person transmission: a study of a family cluster. Lancet 2020;395(10223):514-23.

5. $\mathrm{Su} \mathrm{L}, \mathrm{Ma} \mathrm{X}, \mathrm{Yu} \mathrm{H}$ et al. The different clinical characteristics of corona virus disease cases between children and their families in China - the character of children with COVID-19. Emerg Microbes Infect. 2020;9(1):707-13.

6. Shen Q, Guo W, Guo $\mathrm{T}$, et al. Novel coronavirus infection in children outside of Wuhan, China. Pediatr Pulmonol 2020;55(6):1424-9.

7. Lu X, Zhang L, Du H et al. SARS-CoV-2 Infection in Children. N Engl J Med. 2020;382(17):1663-5.

8. Dong Y, Mo X, Hu Y, Qi X, Jiang F, Jiang Z, Tong S. Epidemiological Characteristics of 2143 Pediatric Patients with 2019 Coronavirus Disease in China. Pediatrics 2020.

9. Meng L, Hua F, Bian Z. Coronavirus Disease 2019 (COVID-19): Emerging and Future Challenges for Dental and Oral Medicine. J Dent Res 2020;99(5):481-7.

10. Lu CW, Liu XF, Jia ZF. 2019-nCoV transmission through the ocular surface must not be ignored. Lancet. 2020;395(10224):e39.

11. Peng $\mathrm{X}, \mathrm{Xu} \mathrm{X}$, Li $\mathrm{Y}$, Cheng L, Zhou $\mathrm{X}$, Ren B.
Transmission routes of 2019-nCoV and controls in dental practice. Int J Oral Sci 2020;12(1):9.

12. Kampf G, Todt D, Pfaender S, Steinmann E. Persistence of coronaviruses on inanimate surfaces and their inactivation with biocidal agents [published correction appears in J Hosp Infect. 2020 Jun 17;:]. J Hosp Infect. 2020;104(3):246-51.

13. American Dental Association. https://www.ada.org/en/science-research/health-policyinstitute/covid-19-dentists-economic-impact. Accessed 20 April 2020.

14. Dental council of India. https://dciindia.gov.in/Admin/NewsArchives/Dental\%20 Clinics\%20Protocols\%20Final.pdf. Accessed 12 May 2020.

15. Ministry of health and family welfare. https://www.mohfw.gov.in/pdf/DentalAdvisoryF.pdf. Accessed 13 August 2020.

16. Ghai S. Teledentistry during COVID-19 pandemic [published online ahead of print, 2020 Jun 16]. Diabetes Metab Syndr. 2020;14(5):933-5.

17. Machado RA, de Souza NL, Oliveira RM, Martelli Júnior H, Bonan PRF. Social media and telemedicine for oral diagnosis and counselling in the COVID-19 era. Oral Oncol 2020;105:104685.

18. Kühnisch J, Ekstrand KR, Pretty I et al. Best clinical practice guidance for management of early caries lesions in children and young adults: an EAPD policy document. Eur Arch Paediatr Dent 2016;17(1):3-12.

19. BaniHani A, Duggal M, Toumba J, Deery C. Outcomes of the conventional and biological treatment approaches for the management of caries in the primary dentition. Int J Paediatr Dent 2018;28(1):12-22.

20. Crystal YO, Niederman R. Evidence-Based Dentistry Update on Silver Diamine Fluoride. Dent Clin North Am. 2019;63(1):45-68.

21. Patil NG, Chan Y, Yan H. SARS and its effect on medical education in Hong Kong. Med Educ 2003;37(12):1127-8. 